\title{
NOUVELLE
}

\section{Contrôle de I'homéostasie glucido-lipidique par les facteurs du cycle cellulaire CDK4 et E2F1}

Pierre-Damien Denechaud, Isabel C. Lopez-Mejia, Lluis Fajas
Département de physiologie, université de Lausanne, rue du Bugnon 7, 1005 Lausanne, Suisse.

pierre-damien.denechaud@unil.ch

isabel.lopezmejia@unil.ch

lluis.fajas@unil.ch
$>$ Chez les mammifères, la régulation du métabolisme glucido-lipidique joue un rôle crucial dans le maintien de l'homéostasie de l'organisme et permet de mieux appréhender les fluctuations des apports énergétiques. Les organes métaboliques, comme le foie et le tissu adipeux, participent au maintien de cet équilibre qui, lorsque perturbé, conduit à l'apparition de pathologies métaboliques comme l'obésité ou le diabète de type 2 [1]. Avec

le cancer, ces

maladies repré-

sentent un pro-

blème de santé

publique majeur

à l'échelle mon-

$(\rightarrow)$ Voir le Faits et chiffres de C. Franc, $m / s$ n' 8-9, août-septembre 2013, page 711 (numéro thématique Diabète : approches thérapeutiques émergentes)

diale $[2,3](\rightarrow)$.

Elles ont également en commun le fait qu'elles impliquent un dysfonctionnement de l'utilisation de l'énergie par les cellules. En effet, les cellules cancéreuses qui prolifèrent nécessitent un certain métabolisme qui va soutenir leurs capacités à se multiplier, notamment lors du passage d'étapes clefs du cycle cellulaire. Ces modifications du métabolisme énergétique des cellules cancéreuses commencent à être bien documentées [4].

Partant de ce postulat, nous nous sommes interrogés sur le rôle que jouent les facteurs du cycle cellulaire dans le contrôle du métabolisme énergétique, et notamment les protéines de la voie CDK4-Rb-E2Fl (cyclin-dependent kinase 4 - protéine du rétinoblastome - E2F transcription factor 1) [5]. Le facteur de transcription $\varepsilon 2 F 1$ et ses régulateurs, la protéine du rétinoblastome $\mathrm{Rbl}$ et la kinase dépendante des cyclines CDK4, sont bien décrits dans la littérature pour leur rôle dans la progression du cycle cellulaire $[6,7]$. Néanmoins, l'étude de modèles de souris invalidées pour CDK4 ou E2Fl nous a permis d'identifier récemment leur importance dans le contrôle du métabolisme glucido-lipidique du foie et du tissu adipeux $[8,9]$.

\section{Le facteur de transcription E2F1 et le métabolisme hépatique}

\section{E2F1 contrôle la glycolyse \\ et la lipogenèse hépatique}

Au sein de l'organisme, le foie joue un rôle majeur dans le maintien de l'homéostasie énergétique en assurant la synthèse de novo d'acides gras à partir du glucose par la voie de la lipogenèse en période post-prandiale. L'induction de l'expression des enzymes de la glycolyse (glucokinase [Gck] et liver pyruvate kinase $[\mathrm{Pk} \mid \mathrm{r}]$ ) et de la lipogenèse (acétyl-CoA carboxylase [Acaca], fatty acid synthase [Fasn] et stearoyl-CoA desaturase [Scdl]) fait intervenir plusieurs déterminants moléculaires dont les principaux sont les facteurs de transcription ChREBP (carbohydrate-responsive elementbinding protein) et SREBP-lc (sterol regulatory element-binding transcription factor $1 c$ ), médiateurs respectifs de l'action du glucose et de l'insuline [10].
L'utilisation de différents modèles de souris transgéniques et d'hépatocytes invalidés pour E2Fl nous a permis de montrer que ce facteur de transcription participe à la régulation de la glycolyse et la lipogenèse hépatique en contrôlant l'expression des gènes impliqués dans ces processus [8]. Des expériences d'immunoprécipitation de chromatine (Chip) ont révélé que les facteurs de transcription ChREBP et SREBP-lc, ainsi que les enzymes de la lipogenèse de novo, sont des cibles directes de $\varepsilon 2 F l$ (Figure IA). E2Fl fait en effet partie des complexes transcriptionnels nécessaires pour moduler l'expression de ces gènes. Sous l'action de l'insuline, CDK4 phosphoryle $\mathrm{Rbl}$, levant de ce fait son effet répresseur sur $\varepsilon 2 \mathrm{Fl}$, pour induire la transcription de ses gènes cibles.

\section{L'invalidation de $\varepsilon 2 \mathrm{Fl}$ protège de la stéatose hépatique}

La stéatose hépatique est une maladie du foie très fréquente dans nos pays industrialisés. Elle se caractérise par une augmentation de la synthèse de novo de lipides participant à l'accumulation de triglycérides dans le foie [10]. Bien que souvent asymptomatique, cette accumulation de graisses dans les cellules est délétère et peut entraîner une réponse inflammatoire, la stéatohépatite, qui peut par la suite conduire à l'apparition d'une cirrhose et de cancer du foie.

Notre laboratoire a pu observer à la fois dans des modèles de souris soumises à un régime riche en graisse, ou généti- 
A

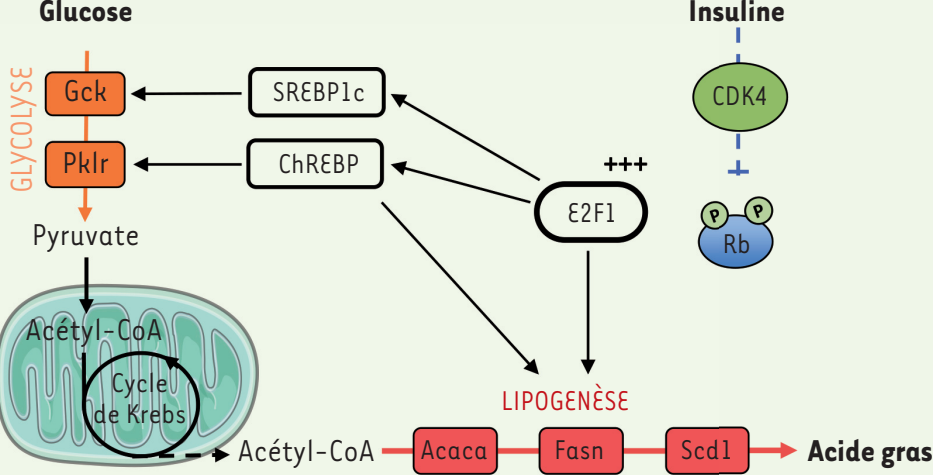

B

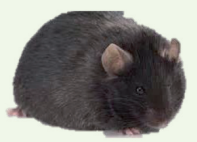

Croisement de souris $\mathrm{db} / \mathrm{db} \times$ ع2F1-/-

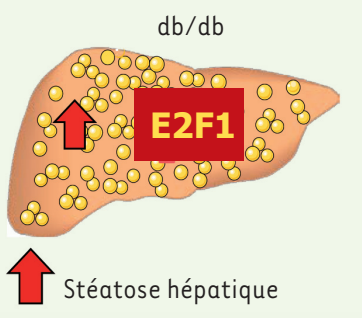

$\mathrm{db} / \mathrm{db} \times \varepsilon 2 \mathrm{Fl} 1-/-$

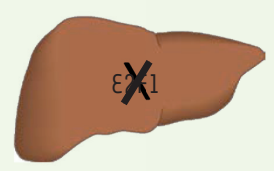

Stéatose hépatique

Figure 1. E2F1 contrôle le métabolisme glucido-lipidique hépatique. A. Le facteur de transcription E2Fl lie directement les promoteurs de ses gènes cibles (Srebplc, ChREBP, Acaca, Fasn, $\mathrm{Scdl}$ ) et contrôle ainsi la voie de la glycolyse et de la lipogenèse dans le foie. Sous l'action de l'insuline, CDK4 phosphoryle Rb et lève ainsi son effet répresseur sur E2Fl. B. L'invalidation de $\varepsilon 2 \mathrm{Fl}$ dans le modèle de stéatose hépatique de souris $\mathrm{db} / \mathrm{db}$ protège de cette pathologie via une diminution de la synthèse de novo d'acides gras. Srebplc: sterol regulatory element-binding transcription factor $1 c$; ChREBP : carbohydrate-responsive element-binding protein ; Acaca: acétyl-CoA carboxylase; Fasn : fatty acid synthase ; Scdl : stearoyl-CoA desaturase ; CDK4 : cyclin-dependent kinase 4; Rb: protéine du rétinoblastome; Gck: glucokinase; Pklr: liver pyruvate kinase.

quement obèses et diabétiques (souris $\mathrm{db} / \mathrm{db}$, déficientes pour le récepteur à la leptine ${ }^{1}$ ), mais également chez l'homme obèse intolérant au glucose, une augmentation de l'activité et de l'expression du facteur E2Fl dans le foie. L'importance de $22 \mathrm{Fl}$ dans cette physiopathologie hépatique a été révélée par le croisement des souris invalidées pour ¿2Fl avec les souris $\mathrm{db} / \mathrm{db}$ précédemment décrites [8]. L'invalidation ou la diminution de l'expression du gène $\varepsilon 2 \mathrm{Fl}$

${ }^{1}$ La leptine est principalement sécrétée par le tissu adipeux blanc et agit au niveau du cerveau (noyau arqué de l'hypothalamus) pour réguler les dépenses énergétiques et la prise alimentaire. chez ces souris obèses améliore considérablement l'accumulation de triglycérides dans le foie et protège ainsi de la stéatose hépatique (Figure 1B).

\section{La kinase CDK4 et le métabolisme adipocytaire}

\section{CDK4 et tissu adipeux}

La kinase CDK4 ne peut être active que sous la forme d'un complexe formé avec une cycline de type D. CDK4, ainsi que deux de ces partenaires, les cyclines D2 et D3, sont exprimées de façon importante dans le tissu adipeux blanc, un tissu avec un taux de prolifération extrêmement faible. Nous avons pu montrer, en utilisant des expériences d'immunofluorescence et de fractionnement cellulaire, que CDK4 se situe dans le cytoplasme des adipocytes, suggérant ainsi une fonction non transcriptionnelle, indépendante de la phosphorylation de $\mathrm{Rbl}$ et donc de ع2F1 [9]. Nous avons utilisé trois lignées de souris transgéniques: des souris $C d k 4^{n c / n c}$, n'exprimant plus la protéine CDK4, des souris $C d k 4^{R 24 C / R 24 C}$, exprimant une forme hyperactive de CDK4, ainsi que des souris $C d k 4^{f l o x} / f l o x$ infectées par des vecteurs viraux exprimant la Cre recombinase $^{2}$, qui n'expriment plus CDK4 spécifiquement dans le tissu adipeux. Chez ces animaux, nous avons pu observer que l'activité de la kinase CDK4 dans le tissu adipeux est positivement corrélée à la masse adipeuse. L'absence de CDK4 conduit à un phénotype «maigre» des souris, corrélé à une diminution de la lipogenèse et une augmentation de la lipolyse du tissu adipeux. À l'inverse, l'activation de CDK4 observée dans le modèle $C d k 4^{R 24 C / R 24 C}$ conduit à une prise de poids corrélée à une augmentation de la lipogenèse et un blocage de la lipolyse du tissu adipeux [9].

\section{CDK4 et signalisation insuline}

Dans le tissu adipeux, l'insuline va se fixer sur son récepteur, qui a une activité tyrosine kinase, et entraîner l'activation d'une voie de signalisation. Cette activation commence par l'autophosphorylation du récepteur à l'insuline, suivie par le recrutement et la phosphorylation des protéines de la famille IRS (insulin receptor substrate). Par la suite, l'activation de la voie de l'insuline requiert l'activation successive de trois kinases, la phosphoinositide 3-kinase (PI3K), la phosphoinositide dependent kinase I (PDKl) et la protéine kinase $B(P K B)$, plus connue

\footnotetext{
${ }^{2}$ Le système de recombinaison Cre-lox utilise l'enzyme recombinase $\mathrm{Cre}$, une tyrosine recombinase issue du bactériophage $\mathrm{Pl}$, afin de cibler des séquences loxP (également issues du bactériophage $\mathrm{Pl}$ ), permettant ainsi d'activer, réprimer, voire même échanger, les gènes situés entre les séquences lox (gènes dits floxés).
} 
A

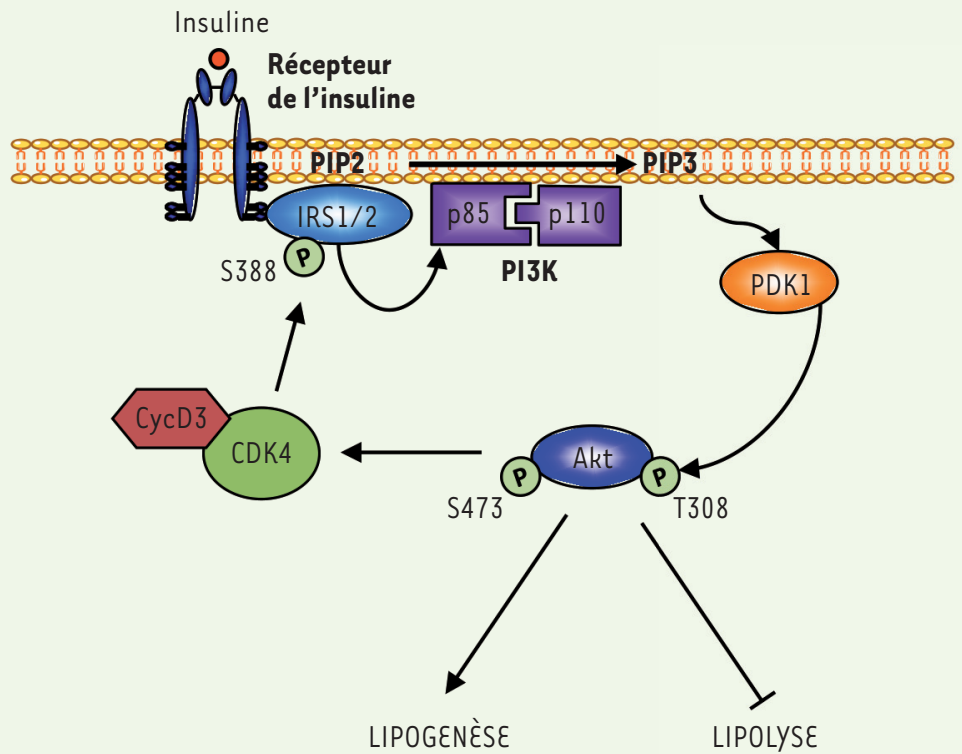

B Rôle de CDK4 dans la physiopathologie de l'obésité

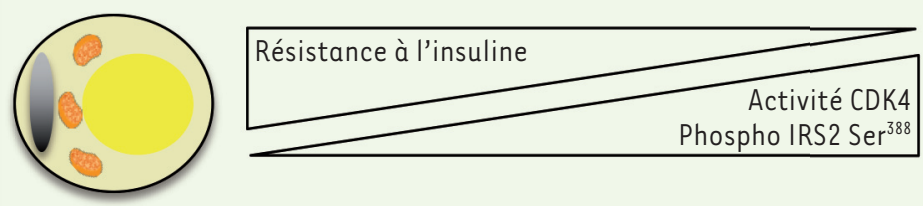

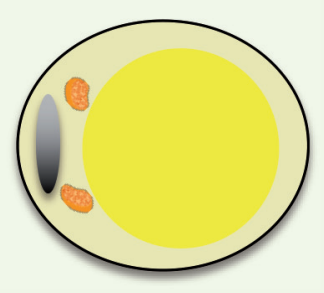

Figure 2. CDK4 contrôle le métabolisme glucido-lipidique adipocytaire. A. L'insuline active CDK4 qui phosphoryle IRS2 sur la Ser ${ }^{388}$ et soutient ainsi la signalisation insulinique active. B. L'activité CDK4 dans le tissu adipeux de personnes obèses est positivement corrélée à la masse adipeuse, ainsi qu'à l'activation de la voie insuline, via la phosphorylation de la Ser 388 de I'IRS2. CDK4 : cyclindependent kinase 4 ; S388, $\mathrm{Ser}^{388}$ : sérine en position 388 ; S473 : sérine en position 473 ; T308: tyrosine en position 308 ; IRS1/2: insulin receptor substrate 1/2; CycD3 : cycline $D 3$; Akt: protéine kinase $B$ PKB ; PI3K : phosphoinositide 3-kinase ; PDKl : phosphoinositide dependent kinase 1 ; PIP2 : phosphatidylinositol-4,5-bisphosphate; $\mathrm{PIP}_{3}$ : phosphatidylinositol-3,4,5trisphosphate. sous le nom d'AKT. Tout comme pour le métabolisme du glucose, les voies de la lipogenèse et la lipolyse sont des voies finement contrôlées par l'insuline dans le tissu adipeux : l'insuline induit la lipogenèse alors qu'elle réprime la lipolyse (Figure 2A).

Afin de mieux comprendre la fonction de CDK4 dans le tissu adipeux, nous avons caractérisé le métabolisme du glucose de ces différents modèles de souris [9]. De manière surprenante, les souris $C d k 4^{\text {R24C/R24C }}$, qui ont une prise de poids plus importante, présentent une amélioration de la sensibilité à l'insuline et de la tolérance au glucose, à l'inverse des souris invalidées pour CDK4 dans le tissu adipeux ( $\left.C d k 4^{\text {flox/flox }}\right)$ qui présentent une sensibilité à l'insuline diminuée. Nous avons également pu mettre en évidence par des expériences de biologie moléculaire que CDK4 est une protéine clef dans la transmission du signal insulinique. En réponse à l'insuline, l'activité de CDK4 augmente et phosphoryle la sérine en position 388 ( $\mathrm{Ser}^{388}$ ) de la protéine IRS2 (insulin receptor substrate 2) pour soutenir l'activation de la voie (Figure 2B).

\section{Activité de CDK4 dans le tissu adipeux de patients obèses}

Notre étude révèle également que l'activité de l'enzyme CDK4 augmente de façon proportionnelle à l'indice de masse corporelle (IMC) dans le tissu adipeux de sujets humains [9]. Même si l'expression de la protéine CDK4 ne change pas entre sujets sains et sujets obèses, l'expression de la cycline D3, ainsi que la phosphorylation de la protéine IRS2 sur la Ser ${ }^{388}$, sont augmentées chez les sujets obèses. De façon intéressante, le niveau de phosphorylation de la Ser ${ }^{388}$ de I'IRS2 est inversement proportionnel à la glycémie à jeun des sujets étudiés, démontrant ainsi une corrélation positive entre l'activité CDK4 et les effets de la voie de l'insuline dans le tissu adipeux.

\section{Perspectives}

Ces travaux mettent en lumière l'intérêt des approches transdisciplinaires, comme ici pour l'étude du cycle cellulaire/cancer et du métabolisme. Ce type d'approche, effectué dans un contexte non prolifératif, montre en effet que des facteurs clés du cycle cellulaire, ici la voie CDK4-Rb-E2Fl, jouent un rôle primordial dans le maintien de l'homéostasie énergétique au niveau du foie et du tissu adipeux $[8,9]$. Par conséquent, ces résultats suggèrent que l'utilisation de médicaments développés pour le cancer pourrait également avoir des effets 
métaboliques et se révéler bénéfiques dans le traitement d'autres pathologies comme l'obésité et le diabète de type 2. $\diamond$

\section{Cell cycle regulators CDK4 and E2F1 control glucose and lipid homeostasis}

\section{LIENS D'INTÉRÊT}

Les auteurs déclarent n'avoir aucun lien d'intérêt concernant les données publiées dans cet article.

\section{RÉFÉRENCES}

1. White MF. Insulin signaling in health and disease. Science 2003 ; 302 : 1710-1.

2. Franc $C$. Le diabète: des chiffres alarmants. Med Sci (Paris) 2013 ; $29: 711-4$.

3. Hanahan D, Weinberg RA. Hallmarks of cancer: the next generation. Cell $2011 ; 144$ : 646-74.

4. Escote X, Fajas L. Metabolic adaptation to cancer growth: from the cell to the organism. Cancer Lett 2015 ; 356 : 171-5.

5. Fajas L. Re-thinking cell cycle regulators: the crosstalk with metabolism. Front Oncol 2013; $3: 4$.
6. Dimova DK, Dyson NJ. The E2F transcriptional network: old acquaintances with new faces. Oncogene 2005 . $24: 2810-26$.

7. Malumbres M, Barbacid M. Mammalian cyclin-dependent kinases. Trends Biochem Sci 2005 ; 30 : 630-41.

8. Denechaud PD, Lopez-Mejia IC, Giralt A, et al. E2FI mediates sustained lipogenesis and contributes to hepatic steatosis. J Clin Invest 2016 ; 126 : 137-50.

9. Lagarrigue S, Lopez-Mejia IC, Denechaud PD, et al. CDK4 is an essential insulin effector in adipocytes. J Clin Invest 2016 ; 126 : 335-48.

10. Denechaud PD, Dentin R, Girard J, Postic C. Role of ChREBP in hepatic steatosis and insulin resistance. FEBS Lett $2008 ; 582$ : 68-73.

\section{NOUVELLE}

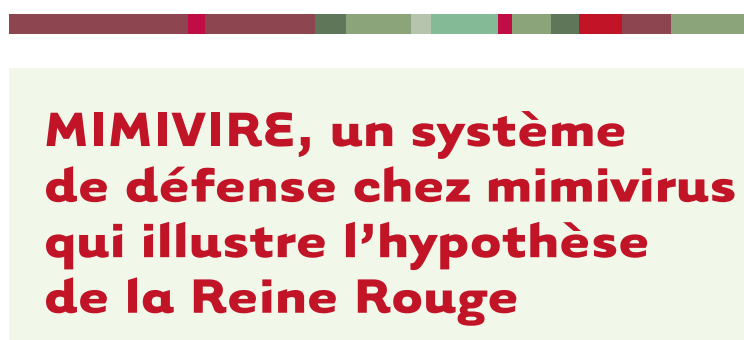

Meriem Bekliz*, Anthony Levasseur ${ }^{\star}$, Bernard La Scola, Didier Raoult

> Les procaryotes sont capables d'acquérir une immunité adaptative contre l'invasion d'éléments génétiques, tels que les plasmides ou les phages, via l'incorporation dans leur génome de courtes séquences d'ADN étranger (de 21 à 72 nucléotides [nt]), système appelé CRISPR-Cas (courtes répétitions palindromiques groupées et régulièrement espacée) [1, 2]. L'acquisition de ces séquences d'ADN étranger survient de façon adjacente aux gènes Cas qui ont pour rôle de cliver I'ADN étranger en fragments de petites tailles. C RIS PR - C a s confère ainsi une résistance face à une seconde infection [14, 15] $(\rightarrow)$. $(\rightarrow)$ Voir le Forum de D. Casane et P. Laurenti, $m / s n^{\circ} 6-7$, juin-juillet 2016, page 640 et la Synthèse de Jacques P. Tremblay, $m / s$ $n^{\circ} 11$, novembre 2015, page 1014 $\varepsilon$ n revanche, les eucaryotes subissent un phénomène analogue à celui des rétrovirus dans le cadre d'une coévolution antagoniste communément appelée «hypo-

thèse de la Reine Rouge » [3]. Le principe de cette hypothèse consiste à considérer qu'une espèce évolue en permanence et ce, dans le but de maintenir son aptitude à lutter contre l'évolution de compétiteurs avec lesquels l'espèce co-évolue dans le cadre d'une course aux armements. À ce jour, le système CRISPR-Cas n'avait été observé que chez les bactéries et les archées

[16] $(\rightarrow)$, mais pas chez des virus $[1,2]$.

$(\rightarrow)$ Voir la Nouvelle de $n^{\circ} 12$, décembre 2014, page 1066

La découverte des virus géants d'amibes, vivant en compétition avec d'autres microbes, a contesté la définition classique d'un virus $[4,5]$. Depuis leur découverte, les virus géants ont révélé des caractéristiques phénotypiques et génotypiques uniques qui vont à l'encontre de la définition classique d'un virus, les plaçant à proximité de certains microbes. Les mimiviruses au diamètre supérieur à 0,5 micromètre, sont aisément visibles avec un simple microscope H. Gilgenkrantz, $m / s$
Aix-Marseille Université, Unité de recherche sur les maladies infectieuses et tropicales émergentes (URMITE), UM63, CNRS 7278, IRD 198, Inserm U1095, IHU Méditerranée Infection, Pôle des Maladies Infectieuses, Assistance Publique-Hôpitaux de Marseille, Faculté de médecine, 27, boulevard Jean Moulin, 13005 Marseille, France.

* Ces auteurs ont contribué également à ce travail.

didier.raoult@gmail.com

bernard.la-scola@univ-amu.fr

optique. Ils possèdent un génome large et complexe qui contient des séquences provenant d'autres organismes [6]. En outre, leur infection possible par des virus, les virophages ${ }^{1}$, qui se multiplient dans l'usine à virus des mimivirus, et la présence des éléments mobiles (transpovirons ${ }^{2}$, polintons ${ }^{3}$ ) ont suscité de vifs débats sur l'origine de ces virus [7, 8]. De plus, les virophages, comme les bactériophages, peuvent s'intégrer au génome de mimivirus sous la forme de provirophage [9].

Un des virophages qui parasite la famille des mimivirus a été baptisé Zamilon [10]. Parmi les trois branches de l'arbre

\footnotetext{
${ }^{1}$ Les virophages, par analogie avec les bactériophages qui sont les virus de bactéries, sont capables d'infecter les virus géants comme le mimivirus.

2 Élément génétique mobile, constitué d’un génome linéaire à $A D N$ d'environ $7 \mathrm{~kb}$ codant 8 gènes, pouvant s'intégrer de façon aléatoire dans le génome de virus.

${ }^{3}$ Les polintons, ou aussi mavericks, sont des éléments d'environ 10-20 kb qui peuvent coder plus de 10 protéines différentes.
} 